\title{
Procesos sociodemográficos y alquileres turísticos en centros históricos. Los casos de Sevilla y Cádiz
}

\author{
Julio-José Parralejo. Universidad de Sevilla, Sevilla, Espańa. \\ Ibán Díaz-Parra. Universidad de Sevilla, Sevilla, España. \\ Belén Pedregal. Universidad de Sevilla, Sevilla, España.
}

RESUMEN | El impacto del Covid-19 ha supuesto una brusca paralización del turismo, generando incertidumbre a nivel internacional. En este marco, el último periodo de auge del sector, correspondiente a la recuperación económica en 2012, y la repentina nueva recesión en 2020, ofrecen un periodo privilegiado para examinar los impactos del turismo en los centros urbanos. Este trabajo analiza la relación entre procesos sociodemográficos y el alquiler turístico en el último periodo de auge del turismo, tomando como caso de estudio los centros históricos de dos ciudades andaluzas de marcado carácter turístico: Sevilla y Cádiz. El análisis se fundamenta en los datos sociodemográficos suministrados por el Censo de Población y Vivienda y el Padrón Municipal de Habitantes, junto a información de alquileres suministrados por distintas fuentes. Como resultado, el trabajo demuestra estadísticamente la relación entre la distribución de los alquileres turísticos y varios procesos sociodemográficos, y su influencia en la pérdida de población.

PALABRAS CLAVE | centros urbanos, gentrificación, transformaciones socioterritoriales.

ABSTRACT | The impact of the Covid-19 has harshly affected tourism, triggering uncertainty at the international level. In this context, the last period of the sector's boom, corresponding to the economic recovery in 2012 and the abrupt new recession in 2020, is a privileged period for examining the impacts of tourism in urban centers. This paper analyzes the relationship between sociodemographic processes and tourist rentals in the last period of the tourism boom, taking as a case study the historical centers of two Andalusian cities of a markedly tourist nature: Seville and Cadiz. The analysis is based on the sociodemographic data provided by the Census of Population and Housing and the Register of Inhabitants, along with rental information provided by different sources. The results statistically demonstrate the relationship of the distribution of tourist rentals with various sociodemographic processes and their influence on the loss of population.

KEYWORDS | urban centers, gentrification, socio-territorial transformations. 


\section{Introducción}

El turismo ha tenido una importancia creciente en España desde la década de los sesenta, proceso que ha llevado a la región a convertirse en una potencia turística (Marchena \& Vera, 1990). Esta tendencia no ha hecho sino incrementarse desde la década de los ańos noventa, particularmente tras la salida de la crisis iniciada en 2008. Tal coyuntura inauguró un nuevo ciclo de aumento tanto del número de visitantes como de la participación del turismo en la economía (Cuadrado-Roura \& López-Morales, 2018), auge alimentado por tendencias previas y algunas innovaciones clave.

El turismo se ha considerado cada vez más como una fórmula milagrosa de atraer recursos económicos a regiones y zonas relativamente pobres del mundo (Capocchi et al., 2019a; Navarrete, 2017). El abaratamiento de los transportes y las innovaciones de gestión, especialmente en el turismo de cruceros y en el transporte aéreo, también han seguido incidiendo en el incremento de los flujos de visitantes entre los distintos rincones del globo. Este proceso ha tenido asimismo, como una de sus características más notables, la irrupción de las tecnologías de la información y las innovaciones referentes al home sharing, junto con la transformación que ha supuesto del mercado de los alquileres turísticos, con plataformas como la conocida Airbnb (Postma \& Schmuecker, 2017; Veiga et al., 2018).

Como resultado de lo anterior, el turismo se ha convertido en la principal fuente de ingresos para muchas ciudades, dando lugar a la profesionalización del sector y al cada vez mayor uso de nuevas tecnologías (Chamorro, 2008). Esto ha permitido ofrecer servicios de alta calidad y posibilitado el acceso a ellos de distintos estratos sociales, además de haber impulsado una fuerte internacionalización de la actividad, que ha ido incrementándose en los últimos ańos. El impacto de los alquileres turísticos temporales y de las plataformas tipo Airbnb, al mismo tiempo que multiplica las posibilidades para los turistas a la hora de visitar ciudades y acceder a un amplio rango de experiencias, ha significado la intensificación del número de visitas tanto a ciudades tradicionalmente turísticas como a otras que no lo eran tanto, conduciendo a ciertos tipos de congestión y a la competencia por el espacio con los alquileres convencionales en las áreas históricas más visitadas. Esto ha coincidido con cierta alarma social en torno a la saturación de visitantes, especialmente en los centros históricos, con especial atención al crecimiento de la fórmula de los alquileres turísticos (Cócola Gant et al., 2016; Gil \& Sequera, 2018), suscitando la cuestión de la necesidad de regulación estatal de estas actividades (Milano, 2018).

Aunque el impacto del Covid-19 ha generado una brusca interrupción de la actividad turística y una situación de incertidumbre a nivel internacional respecto del grado en que el sector pueda continuar o recuperar la importancia que tenía en 2019, las políticas de fomento que surgen ante la incipiente crisis en España -como el Decreto-Ley 2/2020 del 9 de marzo de Mejora y Simplificación de la Actividad Productiva en Andalucía-, siguen apostando por la actividad turística y el sector inmobiliario y de la construcción. En este marco, el último periodo de auge del sector turismo, claramente definido entre el inicio de la recuperación económica en 2012 y la nueva recesión en 2020, supone un periodo privilegiado para examinar los 
impactos de la actividad turística en la economía de cara a una futura recuperación de la misma, y su necesaria regulación en el contexto actual de innovación tecnológica.

El término 'turistificación' ha proliferado en la academia hispanohablante en la última década para describir los impactos negativos de la intensificación de los flujos turísticos, y muy especialmente de los alquileres turísticos sobre áreas urbanas, donde se observa un desplazamiento de la población residente para introducir visitantes que se hospedan en viviendas convertidas en alojamientos turístico (Barrero \& Jover, 2020). El fenómeno de la turistificación se encuentra estrechamente vinculado a los estudios sobre gentrificación en las áreas centrales de las grandes ciudades que venían desarrollándose una década atrás (Cabrerizo et al., 2017; Pavel, 2016). Esta narrativa tiene su origen en gran parte en la noción de gentrificación turística, introducida por primera vez por Gotham (2005) y que ha sido seguida a posteriori por numerosos investigadores (Cócola Gant, 2019a; Füller \& Michel, 2014). La cuestión ha recibido mucha atención desde los estudios urbanos, especialmente en la península ibérica (Morell, 2018; Sequera \& Nofre, 2018) y ha suscitado diversas publicaciones monográficas (Mansilla, 2018) y números especiales en revistas de alto impacto (Pons et al., 2014). El problema de los impactos del turismo ha sido abordado recientemente desde otras perspectivas; por ejemplo, las publicaciones sobre el overtourism o "sobreturismo", que viene recibiendo una notable atención por parte de las publicaciones especializadas (Capocchi et al., 2019b; World Tourism Organization [unWTo], 2018). La principal particularidad de los trabajos que abordan la cuestión desde la perspectiva de la turistificación es su fuerte vínculo con los estudios urbanos y la relación que establecen entre los procesos vinculados al turismo con otros de carácter sociodemográfico que afectan a la estructura socioespacial de la ciudad, como sería la gentrificación clásica, la gentrificación transnacional o los procesos de repoblación y despoblamiento de las áreas centrales de las ciudades (Jover \& Díaz-Parra, 2019; Slater, 2009).

Siguiendo a Clark (2004), la gentrificación referiría el fenómeno de la sustitución de los residentes y usuarios de cierto sector urbano por otros con mayor poder adquisitivo, debido a la inversión realizada en locales y viviendas de un barrio. En esa línea, Cócola Gant (2015) afirma que la mejora comercial en las áreas centrales excluye a los residentes establecidos desde hace mucho tiempo. El principal resultado sociodemográfico identificable de este proceso sería que la población residente de estos sectores aumenta su estatus, mesurable mediante distintos indicadores (Díaz Parra \& Apaolaza, 2020). La noción de gentrificación transnacional, por su parte, puede interpretarse como el proceso protagonizado por nuevos residentes con mayor estatus, procedentes de otros países (Jover \& Díaz-Parra, 2019). Por su parte, la turistificación podría ser tomada como una sustitución protagonizada, en lugar de por nuevos hogares de un estrato social superior a los preexistentes, por turistas y visitantes eventuales (Cócola Gant, 2015). Jover y Díaz-Parra (2019) remarcan esta diferencia entre gentrificación y turistificación, ya que el segundo proceso conduce a cierto despoblamiento de los espacios afectados, en relación al número de población residente. En ese sentido, Cócola Gant (2019b) describe el fenómeno de la gentrificación turística, que se produce cuando el turismo y la gentrificación clásica coexisten, y sugiere que la intensificación de visitantes hace aumentar el 
valor de las propiedades inmobiliarias, tanto comerciales como residenciales, lo que explicaría el interés en promover el aumento del turismo. Por otro lado, el mismo autor destaca la importancia de la gentrificación turística para economías periféricas que dependen del turismo como factor de crecimiento económico, principalmente en el Mediterráneo o América Latina, donde la llegada de consumidores del Norte global complementa la escasa demanda local y abre la puerta a nuevos inversores.

Wachsmuth y Weisler (2018) hablan de un rent gap turístico, la diferencia existente entre los alquileres convencionales y los alquileres turísticos, como motor del proceso de turistificación. Sin embargo, otros autores han señalado la falta de evidencias sobre la relación de los alquileres turísticos con el desplazamiento de población (Horna \& Merantea, 2017). La mayor parte de los trabajos empíricos se han centrado en el impacto sobre el mercado del alquiler (Ortuño \& Jiménez, 2019; Wachsmuth \& Weisler, 2018) o, cuando han tratado sobre la población, lo han hecho mediante estudios de caso, priorizando metodologías cualitativas, entrevistas y trabajo de campo (Cócola Gant \& Gago, 2019). Existe, por tanto, cierta falta de estudios que aporten evidencias sobre la relación entre los alquileres turísticos y dinámicas expulsivas de los residentes, a partir de indicadores estadísticos reproducibles.

En este contexto, el trabajo se plantea con el objetivo principal de estudiar la relación que ha tenido el auge de los alquileres turísticos con diversos procesos sociodemográficos que venían desarrollándose en los centros históricos de las ciudades andaluzas de gran tamaño, como serían la tendencia a la gentrificación de las áreas centrales y al incremento o regresión de la población residente en estos mismos sectores.

Las preguntas de investigación que orientan este objetivo son: ¿Cómo se relaciona el incremento de los alquileres turísticos con las dinámicas sociodemográficas de la ciudad? ¿En qué medida afectan de manera diferencial a los centros históricos? Partimos de la idea de que los alquileres turísticos guardan relación con la ocurrencia de ciertos impactos sociodemográficos, principalmente el despoblamiento, la elitización o gentrificación de las áreas afectadas, y con el establecimiento de residentes extranjeros procedentes de países más ricos en los centros históricos.

Para identificar estas relaciones, se ha diseñado una serie de indicadores que analizan los procesos sociodemográficos y el mercado del alquiler. Se ha observado el comportamiento de estos indicadores para las ciudades de Sevilla y Cádiz y se ha realizado un análisis de regresión clásica basada en ols (Ordinary Least Squares - mínimos cuadrados ordinarios) para identificar los aspectos sociodemográficos que explican los diferentes patrones de localización de los alquileres turísticos en ambas ciudades.

El trabajo se enmarca en un proyecto de investigación sobre los impactos socioespaciales de la turistificación en grandes ciudades andaluzas. Entre estas, los casos de Sevilla y Cádiz son representativos de dos modelos: una ciudad de interior y de mayor tamaño, y una ciudad costera con mayor peso del turismo de cruceros. El trabajo pretende dar cuenta de lo que ocurre en las ciudades andaluzas de gran tamaño y, al mismo tiempo, aportar información de interés que luego pudiera ser comparada con la creciente casuística a nivel internacional sobre esta temática. 
Después de esta introducción, sigue un apartado metodológico, donde se describen los pasos que ha seguido el análisis, fuentes utilizadas y justificación de los distintos indicadores. A continuación se exponen los resultados, donde se explora de forma comparativa y sucesiva, primero, una serie de indicadores sobre viviendas turísticas y usos de la vivienda; y segundo, otra serie de indicadores sociodemográficos. Seguidamente se exponen los resultados de la regresión ols con la distribución de los alquileres turísticos como variable dependiente. El artículo termina con un breve apartado de conclusiones, donde se señala el diferente comportamiento de los alquileres turísticos en los dos casos de estudio.

\section{Metodología}

El estudio se centra en las ciudades de Sevilla y Cádiz, pudiéndose extrapolar también a ciudades latinoamericanas que tienen características parecidas, al compartir idioma, cultura e historia, salvando las distancias sobre todo respecto del tamaño poblacional de las ciudades americanas respecto a las españolas. Sevilla y Cádiz constituyen dos casos de fuerte interés, dado que ambas son ciudades turísticas con centros históricos de grandes dimensiones y con una reconocida historia y evolución en las últimas décadas (Mato, 2005; Villar \& Fernández, 2017), y se han convertido en un importante recurso económico para la economía local.

Sevilla, con una población cercana a los 690.000 habitantes en 2019, es la capital administrativa de Andalucía, la ciudad con la mayor oferta de alquileres turísticos en la región y la segunda ciudad de España, después de Madrid, con la mayor proporción de viviendas residenciales dedicadas a uso turístico en su centro histórico. En el último año ha recibido la visita de casi 3 millones de personas, el 42\% de ellas de nacionalidad espańola y el $58 \%$ extranjeros, con datos de pernoctaciones del $63,88 \%$ para los extranjeros y del 36,12\% para los españoles, sumando casi 6 millones de pernoctaciones. Por su lado, Cádiz, con más de 116.000 habitantes, ocupa un lugar más secundario respecto del número de apartamentos turísticos, pero es el puerto andaluz donde más ha aumentado el turismo de cruceros en los últimos años (Consejería de Turismo y Deporte, 2018). Los visitantes recibidos por la ciudad el último ańo superan los 260.000, predominando en esta ocasión el turismo nacional, con un 56,21\%, respecto al $43,79 \%$ de extranjeros, alcanzando las 630.000 pernoctaciones, con un $44,66 \%$ de extranjeros y un $55,34 \%$ de españoles, según indican los datos extraídos de la Encuesta de Ocupación Hotelera (ЕОH) del Instituto Nacional de Estadística (INE, 2019). La zona de estudio está delimitada por los términos municipales de ambas ciudades y, dentro de estas, los centros históricos, los cuales corresponden en Sevilla al distrito 1 y en Cádiz a los distritos del 1 al 7, incluido (Figura 1).

El análisis busca evidenciar la relación entre la presión del alquiler turístico y distintas variables sociodemográficas. Para ello está dividido en dos partes bien diferenciadas. En la primera se realiza un análisis descriptivo a partir de indicadores sociodemográficos, de vivienda y del mercado del alquiler. En la segunda, un análisis inferencial mediante oLs, tomando como variable dependiente la distribución 
de apartamentos turísticos y varias variables independientes de distintos aspectos sociodemográficos.

FIGURA I | Localización de las viviendas turísticas en los casos de estudio: centros históricos de Sevilla y Cádiz

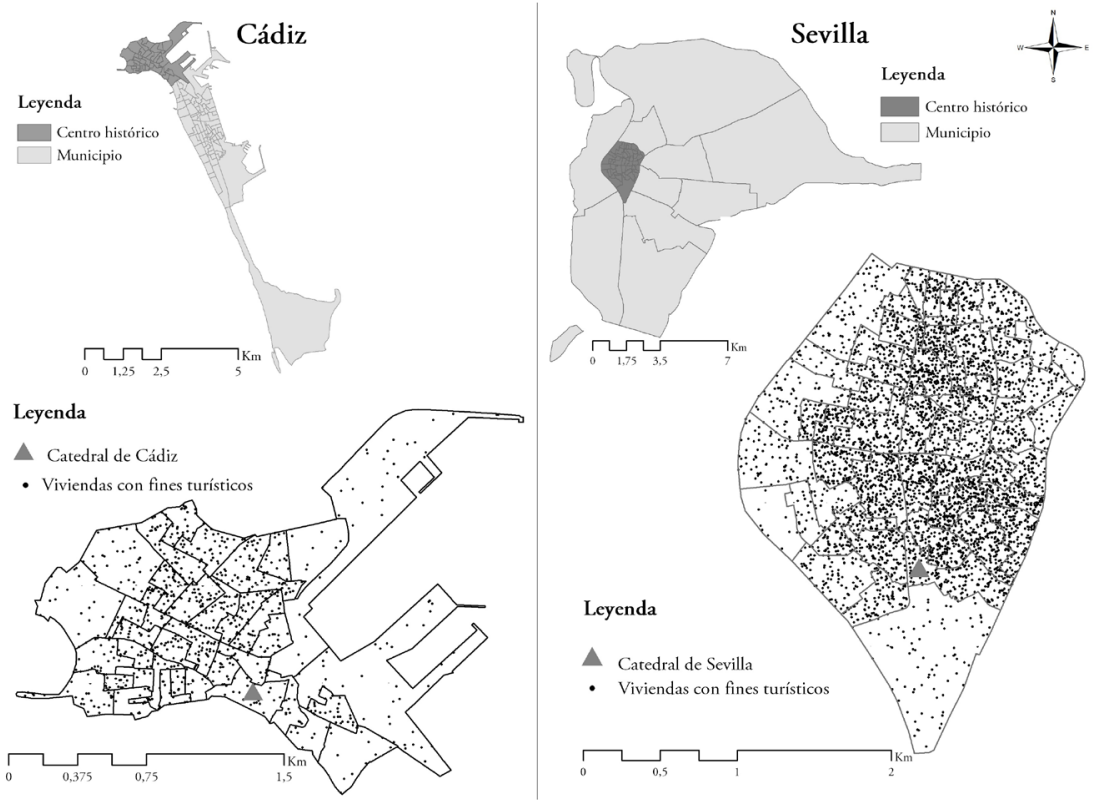

FUENTE: ELABORACIÓN PROPIA A PARTIR DE DATOS ESPACIALES DE REFERENCIA DE ANDALUCÍA (DERA), INSTITUTO DE ESTADÍSTICA Y CARTOGRAFÍA DE ANDALUCÍA Y DATAHIPPO.ORG

Las unidades espaciales utilizadas en el análisis descriptivo son el término municipal de cada una de las ciudades y el centro histórico, tal y como está delimitado en el mapa de la Figura 1. Para el análisis inferencial se ha recurrido a la sección censal. La información sociodemográfica y sobre los usos de la vivienda utilizada procede de los dos últimos Censos de Población y Vivienda realizados en España (2001 y 2011) y los datos anuales de los respectivos registros municipales de población (Padrón Municipal de Habitantes), proporcionados por el Instituto Nacional de Estadística y por el Instituto de Estadística y Cartografía de Andalucía. Los datos censales cuentan con varias limitaciones conocidas, como su escasa periodicidad (diez años en el caso español) y el cambio de las variables observadas en los diferentes años. A esto se suma que el Censo 2011 se nutrió en gran parte de los registros existentes, a los que añadió una muestra estadística para la mayor parte de la información sociodemográfica. Al no ser un censo exhaustivo, gran parte de su información tiene escasa representación en un grado elevado de desagregación, lo que fuerza a reducir el número de variables que se pueden utilizar en la regresión y a tomar una unidad espacial de mayor tamaño -los distritos- para el análisis descriptivo. No obstante, a pesar de estas limitaciones, los censos demográficos aún constituyen 
la única operación estadística fiable que ofrece información abundante sobre los rasgos sociodemográficos de la población residente, referidos a un nivel de detalle espacial suficientemente desagregado para los objetivos de este trabajo. Respecto a los datos referentes al número de habitantes, extranjeros y población envejecida, se han utilizado los datos del Padrón, que, aunque cuentan con menos información sobre la población residente, permiten realizar series temporales de periodicidad anual, ofreciendo datos para el uno de enero de cada año. El ámbito temporal fijado para en el análisis descriptivo viene dado también por esta cuestión. Se ha tomado como primer año de referencia el 2001 por iniciar la primera década del siglo Xxi, coincidiendo además con un año censal. Por su parte, el final de las series de datos ha sido 2019, último año del que se ha publicado la información demográfica de los registros continuos (Padrón).

Por lo que respecta a los datos sobre el mercado de alquiler, no cuentan en la actualidad con un registro público y se deben extraer de distintas fuentes. Aquellos sobre el número de alquileres turísticos se han obtenido de la página web Datahippo (http://datahippo.org), un proyecto colaborativo para ofrecer datos de diferentes plataformas de alquiler turístico (Airbnb, HomeAway, entre otras). De las diferentes plataformas de homesharing se han tomado exclusivamente los datos de Airbnb, por ser la que tiene mayor número de anuncios, evitando la repetición de anuncios en distintas páginas. Se trata de datos de stock sobre el número de alquileres y su localización geográfica, para el año 2019.

Los datos sobre precios medios del alquiler también hacen referencia al año 2019. Para el alquiler turístico se han tomado directamente de la página de Airbnb y para el alquiler convencional, del portal web Idealista.com. Mientras que el dato del alquiler convencional puede tomarse para un año de alquiler, el alquiler turístico cuenta con el obstáculo de la extremada variabilidad de los precios a lo largo del ańo. Esto se ha solventado parcialmente obteniendo la media aritmética de los datos para distintos periodos del año (temporadas alta y baja). Ha sido necesario asumir dos supuestos relevantes para poder comparar ambos tipos de alquiler, según se señalan a continuación.

Primero, que hay tres meses de temporada alta de turismo para ambas ciudades. Este valor trimestral se ha extrapolado a partir del valor del alquiler turístico correspondiente a dos festividades declaradas de Interés Turístico Internacional en ambas ciudades: las dos semanas de carnavales entre febrero y marzo en Cádiz, y la Semana Santa en abril de Sevilla. Aunque la EOH (INE, 2019) indica que en Cádiz los meses de julio y agosto cuentan con un mayor número de pernoctaciones en hoteles, llegando a alcanzar las 77.000; y que, en Sevilla, mayo y octubre son los meses con mayor ocupación, superando las 559.000 pernoctaciones, los precios de los alquileres en los centros históricos se ven mayormente afectados durante las mencionadas fiestas, ya que la Asociación de Hoteles de Sevilla y Provincia (www. hotelesdesevilla.com) afirma que la ocupación hotelera en Semana Santa 2019 fue de un $90 \%$, mientras que en Cádiz, durante los Carnavales, se llegó a alcanzar el 95\% de ocupación, según la Federación de Empresarios de la Hostelería de la Provincia de Cádiz (www.horecacadiz.org). 
El segundo supuesto asume que la vivienda turística estaría alquilada durante todo el mes en los tres meses considerados de mayor ocupación y la mitad del mes en temporada baja. Además, con objeto de comparar los niveles de renta en ambos alquileres (turístico y convencional), se han restringido los datos a aquellos alquileres disponibles con dos habitaciones en ambos tipos, sin diferenciar habitaciones compartidas o sin compartir, ante la falta de información sobre el número de metros cuadrados en el caso del alquiler turístico. Con estos supuestos se ha calculado la renta mensual estimada del alquiler turístico anual, de tal manera que pueda compararse con la del alquiler convencional.

Los indicadores referentes a la tipología de vivienda, régimen de tenencia y mercado de alquiler vienen desglosados a continuación, donde se indica su descripción, cálculo, fuente y unidad espacial para la que se analiza.

Incremento del peso de las viviendas secundarias

Indicador: Incremento porcentual de viviendas secundarias.

Cálculo: [(Vivienda secundaria $\left.2011^{*} 100\right)$ / Vivienda total 2011] - [(Vivienda secundaria $2001 * 100)$ / Vivienda total 2001].

Fuente: Censos de Población y Vivienda (2001-2011).

Unidad Espacial: Municipal, centro histórico.

Infrautilización del parque de viviendas

Indicador: Incremento porcentual de viviendas vacías.

Cálculo: [(Vivienda vacía $\left.2011{ }^{*} 100\right)$ / Vivienda total 2011] - [(Vivienda vacía $2001 * 100)$ / Vivienda total 2001].

Fuente: Censos de Población y Vivienda (2001-2011).

Unidad Espacial: Municipal, centro histórico.

\section{Peso del inquilinato}

Indicador: Incremento porcentual de viviendas en alquiler sobre las viviendas principales.

Cálculo: [(Vivienda alquiler $2011 * 100)$ / Vivienda principal 2011] - [(Vivienda alquiler $2001{ }^{*} 100$ ) / Vivienda principal 2001].

Fuente: Censos de Población y Vivienda (2001-2011).

Unidad Espacial: Municipal, centro histórico.

\section{Centralización de la vivienda turística}

Indicador: Porcentaje de alquileres turísticos en el centro histórico sobre el total de la vivienda de alquiler turístico en el municipio.

Cálculo: (Vivienda alquiler turístico en centro histórico *100) / Total vivienda alquiler turístico en el municipio.

Fuente: Datahippo (2019).

Unidad Espacial: Municipal, centro histórico. 


\section{Turistificación de la vivienda}

Indicador: Porcentaje de alojamientos turísticos respecto del total de alojamientos. Cálculo: (Vivienda alquiler turístico centro histórico *100) / Vivienda total centro histórico.

Fuente: Datahippo (2019) y Censos de Población y Vivienda (2001-2011).

Unidad Espacial: Centro histórico.

\section{Diferencial de renta turística}

Indicador: Diferencia entre el precio medio del alquiler convencional.

Cálculo: [(Renta anual media del alquiler turístico - renta anual media del alquiler convencional) $\left.{ }^{*} 100\right]$ / Renta anual media del alquiler convencional.

Fuente: Airbnb e Idealista (2019).

Unidad Espacial: Centro histórico.

Se trata de indicadores simples que, como se indica, consisten en tasas porcentuales o incrementos porcentuales entre dos ańos de referencia. Junto a estos, a partir de los datos sobre alquiler convencional y turístico, se construye el diferencial de renta turística, que expresa la diferencia entre la renta arrojada durante un año por el alquiler turístico frente al convencional, expresado en el porcentaje de beneficio extra que obtendría un propietario al pasar de un alquiler convencional a un alquiler turístico, entendido esto como proxy al rent gap turístico.

Los indicadores sociodemográficos analizados aparecen descritos de igual manera a continuación.

Incremento de la población

Indicador: Incremento total de la población.

Cálculo: Población residente (2001-2019, número de habitantes).

Fuente: Padrón continuo, INE, 2001-2019.

Unidad Espacial: Centro histórico.

\section{Peso demográfico del centro histórico}

Indicador: Incremento del porcentaje de población municipal en el centro histórico. Cálculo: (Población residente en el centro histórico *100) / Población total en el municipio.

Fuente: Padrón continuo, INE, 2001-2019.

Unidad Espacial: Centro histórico.

\section{Envejecimiento}

Indicador: Incremento porcentual mayores de 65 años.

Cálculo: (Mayores de 65 años *100) / Población total.

Fuente: Padrón continuo, INE, 2001-2019.

Unidad Espacial: Centro histórico. 


\section{Transnacionalización de las áreas centrales}

Indicador: Incremento porcentual de extranjeros residentes en el centro histórico. Cálculo: (Total población extranjera *100) / Población total.

Fuente: Censos de Población y Vivienda (2001-2011). Unidad Espacial: Centro histórico.

\section{Gentrificación}

Indicador: Incremento porcentual de personas con estudios superiores en el centro respecto del incremento en el conjunto de la ciudad.

Cálculo: (Población adulta con estudios universitarios *100) / Población adulta total.

Fuente: Censos de Población y Vivienda (2001-2011).

Unidad Espacial: Municipal, centro histórico.

\section{Gentrificación transnacional}

Indicador: Incremento porcentual de extranjeros de la UE- $15^{1}$ en el centro respecto del incremento en el conjunto de la ciudad.

Cálculo: (Extranjeros de UE-15*100) / Población total.

Fuente: Censos de Población y Vivienda (2001-2011).

Unidad Espacial: Municipal, centro histórico.

La transnacionalización se calcula mediante el porcentaje de población extranjera sobre el total de la residente, y como indicador de la gentrificación transnacional se considera solo el grupo de población extranjera procedente de la UE-15, que, sin contar a España, supondría los 14 países que formaban parte de la Unión Europea antes de 2004. Para el análisis de la gentrificación, se ha tomado como proxy del estatus de la población el nivel de estudios y, en concreto, el porcentaje de adultos con estudios superiores. Este dato adquiere sentido para aproximarse a la noción de gentrificación, en la medida en que analizamos cómo varía entre 2001 y 2019 en el centro histórico en comparación con la variación del mismo dato para el conjunto de la ciudad (Díaz Parra \& Apaolaza, 2020).

Finalmente, se ha realizado una regresión clásica de mínimos cuadrados (ols), tomando como variable dependiente el número de alquileres turísticos ofertados por Airbnb a escala de sección censal. El resultado explicaría un porcentaje de la varianza de esta variable dependiente a partir de la distribución de otra serie de variables independientes, descartando estadísticamente la probabilidad de ausencia de relación entre ellas. El programa utilizado es Geoda, que añade a los test que suelen realizarse en este tipo de modelos, test de autocorrelación espacial (en concreto aquí se ha utilizado la I de Morán), basado en una matriz de pesos espaciales creada a partir de la cartografía censal. Este test sirve básicamente para descartar la existencia de una distribución aleatoria de las variables (Rey et al., 2015).

1 UE-15 se refiere a los países pertenecientes a la Unión Europea antes de 2004, año en el que se amplía el número de países a la ue de los 25. 
El modelo creado ha buscado incluir variables demográficas, de estatus y de vivienda, obtenidas fundamentalmente del Censo y del Padrón, a las que se ha añadido la variable de distancia al centro, obtenida mediante un geoproceso operado en el programa de análisis geográfico Qgis. No obstante, respecto al análisis geoespacial, las variables que se han podido utilizar han sido escasas, debido a los mencionados problemas del Censo de Población de 2011 para trabajar a un elevado grado de desagregación.

\section{Resultados}

A continuación, se presentan los resultados en tres subapartados. Los dos primeros responden al análisis descriptivo de los indicadores de vivienda, mercado del alquiler y sociodemográficos. El análisis empieza mostrando el uso del parque de vivienda en los centros históricos seleccionados (viviendas vacías, secundarias, alquileres), la mayor o menor concentración en los mismos de los alquileres turísticos, y la mayor o menor diferencia de la renta arrojada por el alquiler turístico frente al convencional. Seguidamente, se observa la medida en que una mayor concentración de alquileres turísticos o un mayor diferencial de renta turística coincide en los centros históricos estudiados con procesos de carácter sociodemográfico: despoblamiento y envejecimiento, gentrificación y transnacionalización de la población. Esto permitirá que en el apartado de conclusiones se lancen algunas nuevas hipótesis sobre la naturaleza de esta relación y de las diferencias observadas entre los dos casos de estudio: Sevilla y Cádiz.

Finalmente, en el tercer apartado se analiza en qué medida el uso de la vivienda y las características sociodemográficas pueden explicar la distribución de los alquileres turísticos.

Evolución de la vivienda en los centros históricos de Sevilla y Cádiz

TAbla I | Porcentaje de viviendas secundarias, vacías y en alquiler en los municipios y centros históricos (CH) de Sevilla y Cádiz (2001-2011)

\begin{tabular}{|c|c|c|c|c|c|c|c|c|c|}
\hline & \multicolumn{3}{|c|}{ \% VIVIENDA SECUNDARIA } & \multicolumn{3}{|c|}{ \% VIVIENDA VACÍA } & \multicolumn{3}{|c|}{ \% VIVIENDA EN ALQUILER } \\
\hline & $200 I$ & 20 I I & $\begin{array}{l}200 I- \\
2011\end{array}$ & 2001 & 20 I I & $\begin{array}{c}2001- \\
2011\end{array}$ & 2001 & 20 I I & $\begin{array}{l}200 I- \\
2011\end{array}$ \\
\hline Cádiz & 6,14 & 9,03 & $+2,89$ & 10,36 & 11,62 & $+1,25$ & 31,59 & 24,85 & $-6,74$ \\
\hline Cádiz CH & 3,90 & 6,80 & $+2,91$ & 14,76 & 19,72 & $+4,96$ & 54,99 & 44,90 & $-10,80$ \\
\hline Sevilla & 7,22 & 6,11 & $-1,11$ & 14,71 & 14,29 & $-0,42$ & 10,35 & 11,81 & $+1,46$ \\
\hline Sevilla $\mathrm{CH}$ & 9,09 & 9,10 & $+0,01$ & 29,27 & 24,37 & $-4,90$ & 26,52 & 27,04 & $+0,52$ \\
\hline
\end{tabular}

FUENTE: ELABORACIÓN PROPIA A PARTIR DE DATOS DEL CENSO DE POBLACIÓN Y VIVIENDA 200 I Y 20 I I

La Tabla 1 presenta el peso de las viviendas secundarias, vacías y en alquiler y su variación entre los dos últimos Censos de Población (2001 y 2011). La vivienda secundaria en el municipio de Sevilla representaba en 2001 el 7,22\% del total de viviendas de la ciudad, mientras que en su centro histórico ese porcentaje era mayor, 
superando el 9\%. En Cádiz, al contrario, el dato era superior en el municipio, $6,14 \%$ de viviendas secundarias, mientras que el peso porcentual en el centro histórico era menor al 4\%. Respecto a la vivienda vacía, los dos centros históricos superaban porcentualmente al municipio, teniendo el centro histórico de Sevilla cerca de un 30\% de viviendas vacías y en el de Cádiz, un 14,76\%. Esto implica que el 40\% de viviendas que había en 2001 en el centro histórico de Sevilla no estaban ocupadas todo el año, cifra que en Cádiz se acercaba al 20\%.

Ya en 2011, en la ciudad de Sevilla los datos reflejan una mínima disminución de la proporción de vivienda secundaria, mientras el centro histórico se mantiene estancado, al contrario que en Cádiz, donde la vivienda secundaria aumenta en la ciudad y su centro histórico casi en tres puntos. La vivienda vacía disminuye en Sevilla, siendo llamativa la cifra de cerca de cinco puntos porcentuales que pierde el centro histórico. En Cádiz este dato no varía de manera excesiva en el municipio, pero sí en su centro histórico, aumentando casi en cinco puntos, hasta acercarse a un $20 \%$ del stock de vivienda vacía. Como resultado, el centro histórico de Cádiz cuenta con un mayor porcentaje de viviendas no habitadas de manera continuada, un $26 \%$, mientras que en el centro histórico de Sevilla este dato baja hasta el $23 \%$. En cualquier caso, estos datos demuestran que, en el siglo xxI, en ambos centros históricos existe una infrautilización del parque de viviendas que podían ser utilizadas para el alquiler turístico.

Los datos arrojados por los censos sobre la proporción de viviendas en régimen de alquiler convencional siguen pautas similares, con una evidente concentración en los centros históricos, aunque con mucha mayor importancia tanto en el municipio como en el centro de Cádiz. En Sevilla, el porcentaje de viviendas en régimen de alquiler se incrementa ligeramente en el centro histórico durante el periodo 20012011, aunque menos que en el conjunto de la ciudad. En Cádiz sucede el fenómeno contrario: el alquiler desciende significativamente, perdiendo hasta 10 puntos en el centro histórico. El comportamiento de la vivienda de alquiler en Sevilla se corresponde con el crecimiento demográfico, rejuvenecimiento e incremento de la población extranjera en el centro histórico en la primera década del siglo XXI, como se verá más adelante, poniendo en juego un mayor número de viviendas, muchas en alquiler. En el caso de Cádiz, el mercado inmobiliario en este periodo parece dirigirse en mayor medida a la propiedad, dentro de la cual, como se ve en la Tabla 1 , tiene un peso considerable la vivienda secundaria.

TABLA 2 Turistificación de la vivienda y centralización de la vivienda turística (2019)

\begin{tabular}{|l|c|c|c|}
\cline { 2 - 4 } \multicolumn{1}{c|}{} & $\begin{array}{c}\text { NÚMERo TOTAL DE } \\
\text { ALQUiLeR TURÍstico }\end{array}$ & $\begin{array}{c}\text { \% CENTRALIZACIóN } \\
\text { DEL ALQUILER } \\
\text { TURÍstico }\end{array}$ & $\begin{array}{c}\text { \% TURISTIFICACIÓN } \\
\text { DE LA VIVIENDA }\end{array}$ \\
\hline Centro histórico Cádiz & 897 & 61,61 & 6,51 \\
\hline Centro histórico Sevilla & 4.840 & 65,20 & 18,24 \\
\hline
\end{tabular}

FUENTE: ELABORACIÓN PROPIA A PARTIR DE DATOS DE DATAHIPPO 
Por lo que respecta al número de alquileres turísticos, en la Tabla 2 se han incluido los indicadores desarrollados a partir de los datos de alquileres turísticos publicitados por Airbnb para ambas ciudades, extraídos de la plataforma Datahippo. Como puede observarse, estos indicadores varían mucho entre el centro histórico de Cádiz y el de Sevilla, siendo mucho más elevados en este último, coincidiendo con la mayor superficie y el mayor número de residentes.

El indicador que se ha denominado de centralización de la vivienda turística, que muestra la proporción del total de alquileres turísticos del municipio que se localizan en el centro histórico, muestra un dato similar en ambas ciudades, con un valor por encima del $60 \%$. La denominada turistificación de la vivienda, es decir, el porcentaje de viviendas del centro histórico que están dedicadas a alquiler turístico, muestra un dato mucho más elevado en el caso de Sevilla. Más del 18\% del total de viviendas del centro histórico de Sevilla estaban dedicadas a usos turísticos, frente a solo el 6,51\% del centro histórico de Cádiz.

TABla 3 | Diferencial de Renta Turística, 2019

\begin{tabular}{|c|c|c|c|c|}
\hline CASO DE ESTUDIO & $\begin{array}{c}\text { (A) RENTA } \\
\text { MEDIA ANUAL } \\
\text { TURÍSTICA } \\
\end{array}$ & $\begin{array}{c}\text { (B) RENTA } \\
\text { MEDIA ANUAL } \\
\text { CONVENCIONAL }\end{array}$ & A-B & {$[(\mathbf{A}-\mathbf{B}) * \mathbf{I O O} / \mathbf{A}]$} \\
\hline Sevilla centro histórico & $30.832,68 €$ & $12.943,68 €$ & $17.889 €$ & $58,02 \%$ \\
\hline Cádiz centro histórico & $31.593,24 €$ & $7.872 €$ & $23.721,24 €$ & $75,08 \%$ \\
\hline
\end{tabular}

En la Tabla 3 se muestra el diferencial de renta turística, calculada como se indicaba en detalle en el apartado metodológico. El indicador muestra la diferencia de renta que obtendría un arrendador entre un alquiler convencional y uno turístico, para un apartamento de dos habitaciones en el centro histórico, calculado para un año completo. En el centro de la capital andaluza, el incremento del beneficio bruto arrojado por un departamento de este tipo sería de un 58,02\%, mientras que en el centro histórico de Cádiz sería aún mayor, llegando al 75,08\%. Esta diferencia sería la principal motivación para cambiar alquileres convencionales por alquileres turísticos, o para introducir viviendas vacías en este tipo de mercado. La gran diferencia entre Sevilla y Cádiz puede deberse a la mayor restricción de la oferta en la segunda ciudad, menor número de alquileres turísticos que coincide con un parque de viviendas más intensamente utilizado en el centro histórico, menor peso de las viviendas vacías y mayor peso del alquiler convencional.

\section{Evolución sociodemográfica en los centros históricos}

Durante la segunda mitad del siglo xx, los centros históricos de las ciudades de Sevilla y Cádiz se encontraban en fuerte regresión demográfica. Perdían población, generalmente a favor de otros barrios de sus respectivas ciudades o de sus áreas metropolitanas, al tiempo que envejecían notoriamente. La renovación urbana sobre este tipo de centros históricos, generalizada en las ciudades de cierto tamaño de España, se produjo a partir de la década de los años noventa (Díaz Parra, 2010; Troitińo, 1996; Vinuesa, 1996). 
TABla 4 Cambios en la población de los centros históricos de Cádiz y Sevilla

\begin{tabular}{|c|c|c|c|}
\hline & AÑo & $\begin{array}{c}\text { CENTRO HISTÓRICO } \\
\text { DE SEVILLA }\end{array}$ & $\begin{array}{c}\text { CENTRO HISTÓRICO } \\
\text { DE CÁDIZ }\end{array}$ \\
\hline \multirow{3}{*}{ Población total } & 2001 & 52.840 & 45.271 \\
\hline & 2019 & 56.037 & 34.750 \\
\hline & $\Delta 2001-2019$ & +3.197 & -10.521 \\
\hline \multirow{3}{*}{$\begin{array}{l}\% \text { peso demográfico del } \\
\text { centro histórico }\end{array}$} & 2001 & 7,52 & 32,81 \\
\hline & 2019 & 8,14 & 29,95 \\
\hline & $\Delta 2001-2019$ & $+0,62$ & $-2,86$ \\
\hline \multirow{3}{*}{$\%$ envejecimiento } & 2001 & 20,37 & 17,72 \\
\hline & 2019 & 20,51 & 21,96 \\
\hline & $\Delta 2001-2019$ & $+0,14$ & $+4,24$ \\
\hline \multirow{3}{*}{ \% transnacionalización } & 2001 & 2,49 & 0,55 \\
\hline & 2019 & 6,42 & 2,95 \\
\hline & $\Delta 2001-2019$ & $+3,93$ & $+2,40$ \\
\hline
\end{tabular}

FUENTE: ELABORACIÓN PROPIA A PARTIR DEL REGISTRO MUNICIPAL DEL PADRÓN CONTINUO, INSTITUTO NACIONAL DE ESTADÍSTICA

En el año 2019, la población que vivía en el centro histórico de Sevilla alcanzaba un total de 56.037 habitantes, lo que supone un incremento de más de 3.100 personas con respecto al ańo 2001; en Cádiz ocurre lo contrario: en el mismo periodo pierde más de 10.000 habitantes, quedándose el centro con 34.750 residentes. Podemos observar cómo el centro histórico de Cádiz tiene más peso poblacional que la ciudad de Sevilla, a pesar de haber perdido habitantes y Sevilla de haberlos ganado. Un $29,95 \%$ de la población total de Cádiz sigue viviendo en el centro, mientras que en Sevilla este dato es tan solo un $8,14 \%$ (ver Tabla 4).

FIgURA 2 | Evolución del peso demográfico de la población residente en el centro histórico $(\%)$

Año 2013

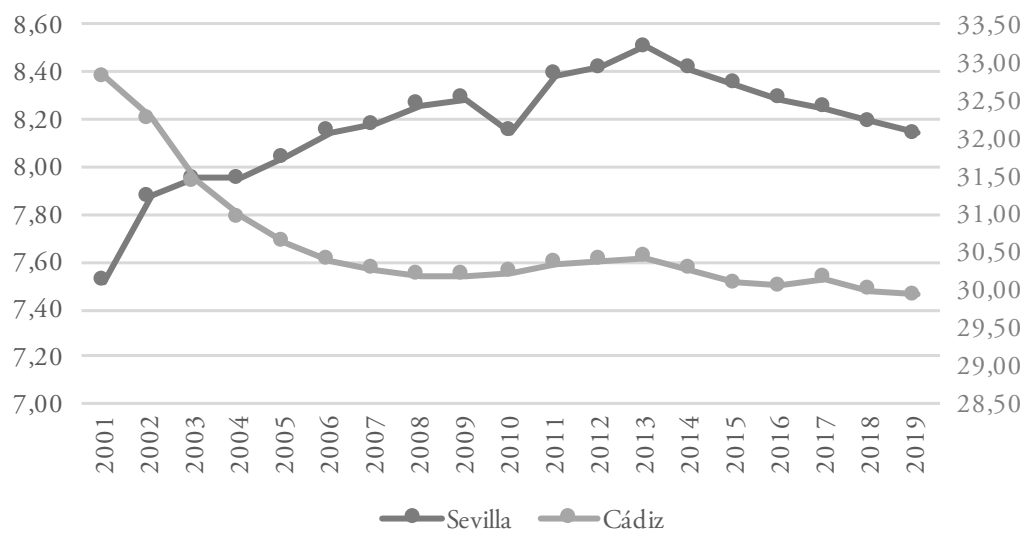

FUENTE: ELABORACIÓN PROPIA A PARTIR DEL REGISTRO MUNICIPAL DEL PADRÓN CONTINUO, INSTITUTO NACIONAL DE ESTADÍSTICA 
El peso de la población del centro histórico en el municipio de Sevilla venía incrementándose desde principios del siglo xxi de forma notable. Esta tendencia se frena y se invierte desde el año 2013, desde el cual se han perdido aproximadamente 3.000 residentes, lo que elimina gran parte del crecimiento anterior. En Cádiz, el peso de la población del centro histórico sobre el total del municipio venía descendiendo a un elevado ritmo hasta 2006, cuando la tendencia se frena, produciéndose incluso un leve incremento del peso entre 2010 y 2013, pero de nuevo a partir de esta fecha el centro histórico vuelve a perder peso (Figura 2).

\section{FIGURA 3 | Evolución del envejecimiento poblacional en los centros históricos}

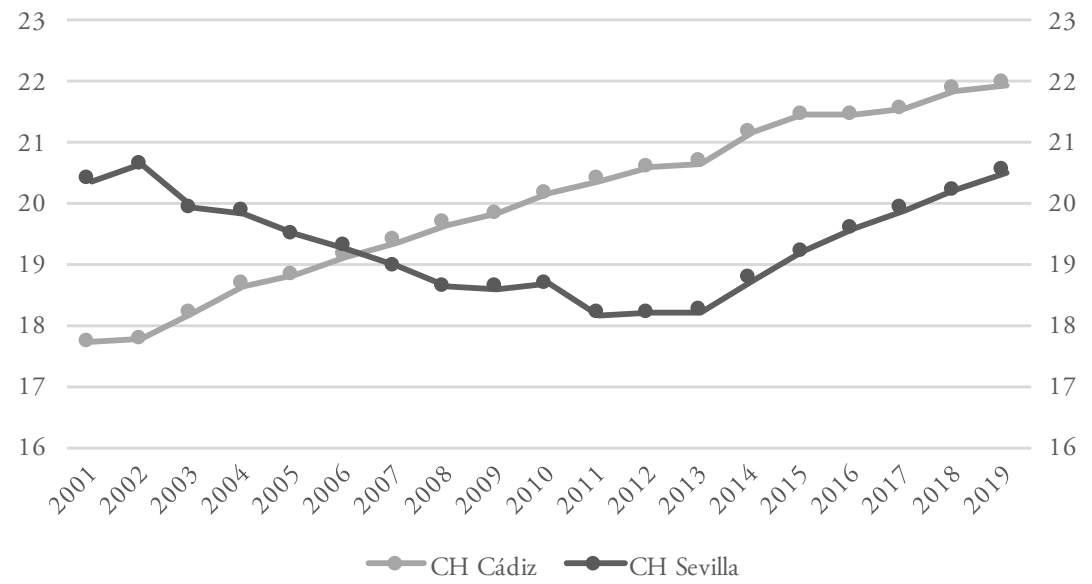

FUENTE: ELABORACIÓN PROPIA A PARTIR DEL REGISTRO MUNICIPAL DEL PADRÓN CONTINUO, INSTITUTO NACIONAL DE ESTADÍSTICA

Respecto a la evolución del envejecimiento poblacional, los datos apuntan a que ambos centros históricos se encuentran muy envejecidos. En el caso de Sevilla, un $20 \%$ de la población de su centro histórico es mayor de 65 años, una proporción que apenas varía entre 2001 y 2019. En Cádiz la proporción de adultos mayores superaba el $17 \%$ en 2001, aumentando más de cuatro puntos en 2019, para acercarse al 22\%. La Figura 3 muestra esta evolución año a año. Aunque aparentemente la proporción de personas mayores no ha variado mucho en el centro histórico de Sevilla, observando el gráfico vemos cómo dicha cifra se redujo en varios puntos porcentuales entre 2001 y 2012, al mismo tiempo que este sector ganaba población total y peso demográfico en el conjunto de la ciudad. En Cádiz, por el contrario, el incremento del envejecimiento ha sido constante desde 2002 (Figura 3).

Finalmente, se ha producido un incremento del peso de la población extranjera notable en ambos centros históricos (Tabla 4). Ambos partían de valores muy bajos en 2001, menores en Cádiz. Desde entonces hasta 2019, en el centro histórico de Sevilla el porcentaje de extranjeros aumenta casi cuatro puntos y el de Cádiz en dos y medio. 
En síntesis, en el caso de Sevilla se produce un claro cambio en las tendencias demográficas a partir de 2013, proceso mucho más atenuado en el centro histórico de Cádiz. En el periodo previo a 2013, en el centro histórico de Sevilla se dio un elevado crecimiento de la población residente, incrementando el peso de la población extranjera y rejuveneciendo al conjunto de la población. En el caso de Cádiz la tendencia es similar, pero este proceso solo se produce a partir de 2005 y de forma mucho más tímida. El decrecimiento de la población residente a partir de 2013 en el centro histórico de Sevilla (Figura 2) implica un nuevo envejecimiento, lo que ilustra que la población que se está perdiendo en ese periodo es población joven.

TABLA 5 | Evolución de la gentrificación en los centros históricos

\begin{tabular}{|l|c|c|c|c|c|}
\cline { 2 - 6 } \multicolumn{1}{c|}{} & AÑo & $\begin{array}{c}\text { CENTRO } \\
\text { HISTÓRICO } \\
\text { DE SEVILLA }\end{array}$ & $\begin{array}{c}\text { MUNICIPIO } \\
\text { DE SEVILLA }\end{array}$ & $\begin{array}{c}\text { CENTRO } \\
\text { HISTÓRICO } \\
\text { DE CÁDIZ }\end{array}$ & $\begin{array}{c}\text { MUNICIPIO } \\
\text { DE CÁDIZ }\end{array}$ \\
\hline \multirow{2}{*}{$\begin{array}{l}\text { \% población adulta con } \\
\text { estudios universitarios }\end{array}$} & 2001 & 28,06 & 15,15 & 9,34 & 14,09 \\
\cline { 2 - 6 } & 2011 & 42,39 & 22,03 & 14,02 & 18,85 \\
\cline { 2 - 6 } & $\Delta 2001-2011$ & $+14,33$ & $+6,88$ & $+4,68$ & $+4,76$ \\
\hline \multirow{2}{*}{$\begin{array}{l}\% \text { extranjeros procedentes } \\
\text { de UE-15 }\end{array}$} & 2001 & 0,82 & 0,23 & 0,15 & 0,44 \\
\cline { 2 - 6 } & 2019 & 2,67 & 0,59 & 0,88 & 0,49 \\
\cline { 2 - 6 } & $\Delta 2001-2019$ & $+1,85$ & $+0,36$ & $+0,73$ & $+0,05$ \\
\hline
\end{tabular}

FUENTE: ELABORACIÓN PROPIA A PARTIR DE LOS CENSOS DE POBLACIÓN Y VIVIENDA 200 I Y 2 OI I Y DEL REGISTRO MUNICIPAL DEL PADRÓN CONTINUO, INSTITUTO NACIONAL DE ESTADÍSTICA

Los datos de la Tabla 5 añaden un matiz a las observaciones anteriores sobre el cambio poblacional en los dos centros históricos objeto de estudio. La población que ha promovido el crecimiento y rejuvenecimiento durante ciertos periodos ha sido de estatus relativamente alto, lo que indica cierto grado de gentrificación e incluso cierto grado de gentrificación transnacional. El centro histórico de Sevilla aumenta más del doble su población con estudios universitarios en comparación con el total del municipio, alcanzando un 42,39\% de personas con dicho rango. En Cádiz no ocurre lo mismo y el municipio en su conjunto tiene mayor peso de personas con estudios superiores que el casco antiguo; el crecimiento ha sido muy parejo tanto en la ciudad como en el centro histórico, pero los datos reflejan que el centro histórico de Cádiz no es y no parece tender a ser un espacio privilegiado de la ciudad, muy al contrario que en el caso de Sevilla. En comparación con los valores nacionales y regionales, los municipios de Sevilla y Cádiz presentan valores superiores a los de referencia. Así, en España, en 2001 el 13,20\% de la población tenía estudios superiores, siendo en Andalucía este dato de un 11,10\%. En 2011 el dato nacional aumentó hasta el 17,90\% y el andaluz alcanzó el 14,60\% de la población con estudios superiores.

Respecto a los extranjeros procedentes de la Unión Europea (UE-15, menos España), el último censo indica que en España residían 933.714 extranjeros (1,97\% de la población española), de los que 174.218 lo hacían en Andalucía (2,06\% sobre el total de la población residente en Andalucía). En 2001 Sevilla registraba un $0,82 \%$ de estos extranjeros respecto al total de la población en el centro histórico, mientras que en la ciudad el dato era menor, con un 0,23\%. En 2019 sí observamos 
un crecimiento considerable de este grupo de extranjeros, alcanzando un 2,67\% de la población residente en el centro histórico, siendo la variación mínima para el conjunto del municipio. Por su parte, Cádiz también presenta unos datos bajos en 2001, aunque ligeramente superiores en el municipio que en el casco antiguo. Sin embargo, en este caso sí se invierte la situación, con un crecimiento notable de la tasa en el centro histórico, mientras que en el conjunto del municipio la variación es escasa. Aun así, la población con este origen no llega a alcanzar el 1\% de la población del centro histórico.

En definitiva, los datos indican una tendencia a la gentrificación del centro histórico en Sevilla que no parece darse en Cádiz (al menos a la fecha del último censo), mientras que la población procedente de los países más desarrollados de la Unión Europea que adoptan su residencia en estas ciudades tiende a elegir los centros históricos para instalarse.

\section{Localización de alquileres turísticos y características sociodemográficas del territorio}

A continuación, la Tabla 6 y la Tabla 7 explican la distribución de los alquileres turísticos por secciones censales, con una clara concentración en las áreas centrales, como se ha visto en el apartado de evolución de la vivienda en los centros históricos de Sevilla y Cádiz con relación a las características sociodemográficas del territorio. En su elaboración, se parte de la hipótesis de que existe una relación estadísticamente significativa entre los aspectos sociodemográficos característicos de la gentrificación y la distribución de los apartamentos turísticos de Airbnb. La variable dependiente es la distribución de los apartamentos turísticos, que se busca predecir mediante un modelo explicativo que combina la distancia al centro de la ciudad, el tipo de uso de la vivienda (viviendas vacías, secundarias y en alquiler convencional) y las características sociodemográficas del espacio (envejecimiento, elitización y transnacionalización).

En el análisis ols, el coeficiente de determinación R2 establece la calidad del modelo para predecir el comportamiento de la variable dependiente. El valor R2 de los modelos que se han aplicado indica que estos son estadísticamente significativos, y tiene un buen nivel de ajuste en ambos casos. En el caso de Cádiz, la R2 ajustada ofrece un dato de 0,62 , lo que se considera un ajuste alto. Podría decirse que las variables independientes explican un $62 \%$ de la varianza de la distribución de los alquileres turísticos ofrecidos por Airbnb. En Sevilla, la R2 es 0,56, lo que implica un ajuste similar. Además, el modelo oLs realizado con Geoda aplica automáticamente varios test de diagnóstico de la regresión y de dependencia (índices de asociación espacial). El dato de multicolinealidad para ambos casos es muy bajo (inferior a 30 a criterio de Rey et al., 2015), lo que no invita a sospechar de una correlación entre las variables explicativas lo suficientemente alta como para llevar a confundir sus efectos marginales. Respecto de la asociación espacial, en ambos modelos se puede descartar la hipótesis nula de una distribución espacial aleatoria de las variables. El modelo de Cádiz cuenta con una I de Moran de 0,35 y en Sevilla, de 0,25, lo que implicaría una autocorrelación espacial moderada entre las variables, es decir, una cierta tendencia a la concentración de valores similares. 
TABLA 6 | Modelo de regresión para Cádiz (oLS)

\begin{tabular}{|c|c|c|c|c|}
\hline $\begin{array}{c}\text { VARIABLES } \\
\text { INDEPENDIENTES }\end{array}$ & \multicolumn{2}{|c|}{ COEFFICIENT } & T-STATISTIC & PROBABILITY \\
\hline CONSTANT & \multicolumn{2}{|c|}{12,2181} & 3,22251 & 0,00198 \\
\hline Población UE-15 & \multicolumn{2}{|c|}{0,396278} & 3,28355 & 0,00164 \\
\hline Estudios superiores & \multicolumn{2}{|c|}{$-4,35372 \mathrm{e}-005$} & $-2,14026$ & 0,03603 \\
\hline Viviendas vacías & \multicolumn{2}{|c|}{0,0331544} & 2,00541 & 0,04902 \\
\hline Viviendas secundarias & \multicolumn{2}{|c|}{0,049972} & 3,57138 & 0,00067 \\
\hline Viviendas en alquiler & \multicolumn{2}{|c|}{0,0428535} & 3,42335 & 0,00107 \\
\hline Distancia al centro & \multicolumn{2}{|c|}{$-0,0044042$} & $-4,3428$ & 0,00005 \\
\hline \multicolumn{5}{|c|}{$\begin{array}{l}\text { R-squared: } 0.622834 \\
\text { Adjusted R-squared: } 0.588546 \\
\text { Akaike info criterion: } 541.047 \\
\text { F-statistic: } 18.1649 \\
\text { Prob(F-statistic): } 2.5368 \mathrm{e}-012\end{array}$} \\
\hline \multicolumn{2}{|c|}{$\begin{array}{l}\text { MULTICOLLINEARITY } \\
\text { CONDITION NUMBER } \\
\end{array}$} & \multicolumn{3}{|c|}{8,183077} \\
\hline \multicolumn{2}{|l|}{ TEST } & $\mathrm{DF}$ & VALUE & PROB \\
\hline \multicolumn{2}{|l|}{ Jarque-Bera } & 2 & 2.2175 & 2997 \\
\hline \multicolumn{2}{|c|}{$\begin{array}{l}\text { TEST AUTOCORRELACIÓN } \\
\text { ESPACIAL }\end{array}$} & $\mathrm{MI} / \mathrm{DF}$ & VALUE & PROB \\
\hline \multicolumn{2}{|l|}{ Moran's I (error) } & 0,3537 & 4,2902 & 0002 \\
\hline
\end{tabular}

FUENTE: ELABORACIÓN PROPIA

TABLA 7 Modelo de regresión para Sevilla (oLS)

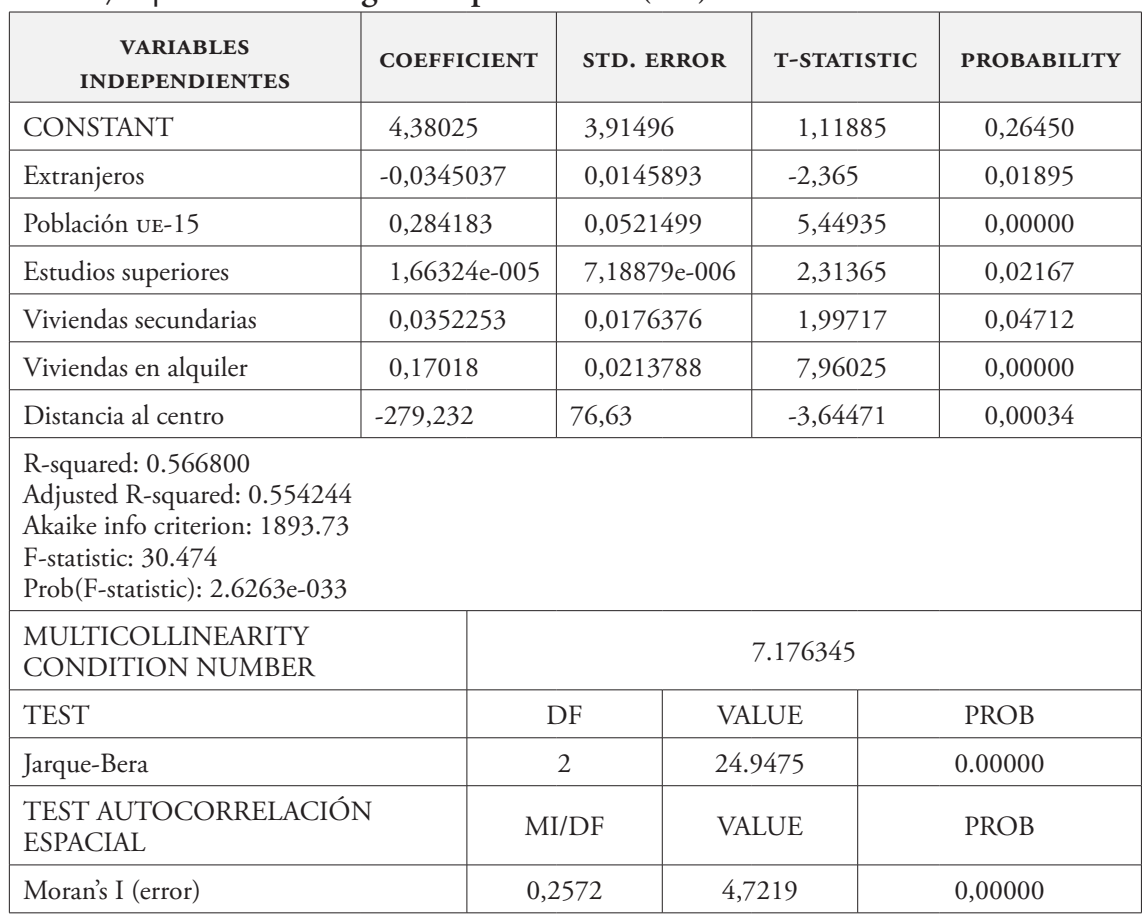

FUENTE: ELABORACIÓN PROPIA 
En ambos casos, la variable sociodemográfica que más contribuye al modelo explicativo es la presencia de residentes extranjeros procedentes de la UE-15, con una relación positiva (a mayor número de residentes extranjeros de la UE, mayor número de alquileres turísticos) y de similar intensidad en los dos casos, Sevilla y Cádiz. En Cádiz, esta es la variable que más contribuye al modelo explicativo, pero es superada por la variable distancia al centro en el caso de Sevilla. El otro aspecto sociodemográfico con peso en el modelo para ambas ciudades sería la presencia de población con estudios superiores, una relación positiva en Sevilla, pero negativa en Cádiz, mientras que en el resto de variables sociodemográficas no son significativas estadísticamente, a unos niveles de significación de 0,05.

La concentración del turismo en el centro histórico de la ciudad es mucho más notable en el caso de Sevilla en comparación con Cádiz, donde tradicionalmente ha existido una carga turística relacionada con las playas occidentales de la ciudad extramuros (Jiménez, 1995). Esto también se reflejaba en el análisis de clústeres. Además, se observa cómo la relación con los patrones de segregación socioespacial, propios de cada ciudad, es mucho más fuerte en el caso de Sevilla, donde el centro de la ciudad se encuentra por completo dentro de las zonas privilegiadas, mientras que los grupos más desfavorecidos, incluidos los extranjeros procedentes de países menos ricos, se concentran invariablemente en la periferia obrera, donde no se ofertan alquileres turísticos. Esta situación no se produce de manera tan clara en Cádiz, debido precisamente al atractivo turístico que supone la existencia de playas fuera del centro histórico.

Los datos de las características de las viviendas son estadísticamente significativos, con la excepción del dato de las viviendas vacías en Sevilla. La relación es positiva en Cádiz y más fuerte en el caso de las viviendas secundarias (a mayor presencia de viviendas secundarias, mayor presencia de alquileres turísticos), menos en el de las viviendas de alquiler y vacías. En el caso de Sevilla, por el contrario, la relación más fuerte y positiva es con el peso de las viviendas de alquiler. Algunos de estos elementos pueden estar más vinculados con la mayor relación del turismo con el centro de la ciudad en el caso de Sevilla, donde -como ya se refirió anteriormentetienden a concentrarse históricamente las viviendas en alquiler, frente a las distintas periferias con un mayor peso de la propiedad. La relación de la distribución con zonas con alta densidad de alquileres tiene también la lógica de la posibilidad de que estos alquileres convencionales se conviertan en alquileres turísticos ante un aumento de la demanda de los mismos. Asimismo, la presencia de viviendas secundarias también puede tener que ver con zonas habituadas a recibir visitantes.

\section{Discusión y conclusiones}

Volviendo a las preguntas iniciales sobre cómo se relaciona el incremento de los alquileres turísticos con las dinámicas sociodemográficas de la ciudad y en qué medida afectan de manera diferencial a los centros históricos, el análisis realizado en ambos casos de estudio demuestra que el incremento de los alquileres turísticos hasta 2019 tiene una relación directa con diversos fenómenos y estructuras demográficas. A nivel estadístico, existe una relación estadísticamente significativa entre 
las estructuras sociodemográficas y de usos de la vivienda de ambas ciudades y la distribución de los alquileres ofertados en Airbnb, siendo las variables más explicativas la proximidad al centro, los usos de la vivienda y la presencia de población extranjera procedente de países de alto nivel socioeconómico.

Asimismo, se ha mostrado cómo existe un cambio de tendencia en las dinámicas demográficas básicas de los centros históricos estudiados a partir de 2013, mucho mayor en Sevilla que en Cádiz. Coinciden al respecto una clara inversión en Sevilla de las dinámicas previas al crecimiento y rejuvenecimiento de su centro histórico, y la irrupción de las plataformas digitales de alquileres turísticos, según ya han planteado autores como Veiga et al. (2018) y Postma y Schmuecker (2017). Ello da fuerza a la hipótesis de que el elevado número de viviendas turísticas y la diferencia de renta arrojada entre la vivienda turística y el alquiler convencional es un motor del proceso de turistificación, como bien indicaban Wachsmuth y Weisler (2018), lo que sería la causante del declive demográfico que se produjo entre 2013 y 2019 -como también apuntaban Barrero y Jover (2020)-, desplazando a la población del centro de la ciudad.

La elevada turistificación de la vivienda en Sevilla coincide con cierto grado de gentrificación transnacional, lo que parece, en primera instancia, una relación lógica entre la presencia de residentes extranjeros procedentes de países más ricos y la oferta de alojamientos temporales para visitantes de estos mismos países, consecuencia ya planteada por Jover y Díaz-Parra (2019). En Sevilla, el fenómeno de turistificación como sustitución de residentes por turista es más claro, pero Cádiz tiene algunos elementos de mayor vulnerabilidad: principalmente, el hecho de que su centro histórico aloja a una población de menor estatus y con un mayor peso de los inquilinos, lo que reduciría su capacidad de competir por el espacio residencial de la zona y aumentaría el riesgo de un desplazamiento involuntario.

La información aportada en este trabajo contribuye a reforzar la idea de que la relación entre gentrificación y turistificación se muestra como un aspecto complejo. De partida, existe una relación significativa entre la presencia de alquileres turísticos, un estatus elevado de la población y la mayor presencia de extranjeros procedentes de países ricos (UE-15 en nuestro caso). Sin embargo, la gentrificación en términos generales, asociada al rejuvenecimiento de los centros históricos en el periodo inmediatamente anterior al impacto de los alquileres turísticos (2013), podría verse afectada negativamente por la pérdida de residentes, principalmente hogares jóvenes, como se ha visto. Si la gentrificación ha tenido relación con el rejuvenecimiento y reversión del declive demográfico de los centros históricos a principios del siglo XXI -algo que parece evidente en el caso de Sevilla-, la turistificación podría estar eliminando estos pequeños logros, lo que no refuta otros aspectos fundamentalmente negativos de la gentrificación.

Existen evidencias suficientes para considerar el impacto demográfico negativo de la concentración de alquileres turísticos en los centros históricos. Esto no se produce en todas las ciudades, ni mucho menos con la misma intensidad; sin embargo, sí encontramos evidencias claras en el caso de Sevilla, con gran afluencia turística a través de vuelos baratos directos desde distintos puntos de Europa. En el momento actual, en el que los países afectados por la crisis global del coronavirus comienzan a 
elaborar sus planes de recuperación económica, España, y en particular Andalucía, parecen apostar fuertemente por la revitalización inmediata de la actividad turística. Teniendo esto en cuenta, los planes de fomento del turismo urbano que se están desarrollando en estas y otras ciudades deben tener en consideración los efectos negativos de un turismo excesivo y, en particular, de algunos tipos de turismo sobre otros, como el propiciado por los alquileres turísticos de plataformas como Airbnb.

\section{Referencias bibliográficas}

Barrero, M. \& Jover, J. (2020). Paisajes de la turistificación: una aproximación metodológica a través del caso de Sevilla. Cuadernos Geográficos, 60(1), 13-34. https://doi. org/10.30827/cuadgeo.v60i1.13599

Cabrerizo, C., Sequera Fernández, J. \& Bachiller, P. G. (2017). Entre la turistificación y los espacios de resistencia en el centro de Madrid: Algunas claves para (re) pensar la ciudad turística. Ecología Política, (52), 78-82. https://doi.org/10.2307/26333515

Capocchi, A., Vallone, C., Amaduzzi, A. \& Pierotti, M. (2019a). Is 'overtourism' a new issue in tourism development or just a new term for an already known phenomenon? Current Issues in Tourism, O(0), 1-5. https://doi.org/10.1080/13683500.2019.1638353

Capocchi, A., Vallone, C., Pierotti, M. \& Amaduzzi, A. (2019b). Overtourism: A literature review to assess implications and future perspectives. Sustainability, 11(12), 1-18. https://doi.org/10.3390/su11123303

Chamorro, R. (2008). Una revolución en el turismo gracias a las Tic. Revista Bit, 170, 30-33.

Clark, E. (2004). The order and simplicity of gentrification: A political challenge. The Gentrification Reader, 24-29. https://doi.org/10.4324/9780203392089

Cócola Gant, A. (2015). Boletín sobre el turismo responsable. Turismo y Desarrollo, 15, 12.

Cócola Gant, A. (2019a). Gentrificación y desplazamiento: desigualdad urbana en ciudades del capitalismo tardío: Manual de Geografía Urbana. En Manual de Geografía Urbana (pp. 297-310). https://doi.org/10.4337/9781785364600.00030

Cócola Gant, A. (2019b). Gentrificación turística. Turistificación Global, (oct. 2019), 291-308.

Cócola Gant, A., Durán, G. \& Janoschka, M. (2016). La ciudad del siglo xxi: políticas públicas urbanas, desplazamientos y contestaciones. Íconos - Revista de Ciencias Sociales, (56), 11. https://doi.org/10.17141/iconos.56.2016.2351

Cócola Gant, A. \& Gago, A. (2019). Airbnb, buy-to-let investment and tourism-driven displacement: A case study in Lisbon. Environment and Planning A: Economy and Space, 1-18. https://doi.org/10.1177/0308518X19869012

Consejería de Turismo y Deporte. (2018). Turismo de cruceros en Andalucía. Consejería de Turismo y Deporte, Sevilla.

Cuadrado-Roura, J. R. \& López-Morales, J. M. (2018). El turismo en la recuperación y el equilibrio exterior de España. Papeles de Economía Española, (febrero), 154-171. http://www.albayan.ae

Díaz Parra, I. (2010). Cambios en la geografía social de Sevilla 1981-2001. Cuadernos Geográficos, (46), 139-161. https://doi.org/10.30827/cuadgeo.v46i0.634 06 
Díaz Parra, I. \& Apaolaza, R. (2020). Una propuesta metodológica para identificar gentrificación a partir de los censos de población. Estudios Demográficos y Urbanos, 35(3), 629-661. http://dx.doi.org/10.24201/edu.v35i3.1883

Füller, H. \& Michel, B. (2014). "Stop being a tourist!" New dynamics of urban tourism in Berlin-Kreuzberg. International Journal of Urban and Regional Research, 38(4), 13041318. https://doi.org/10.1111/1468-2427.12124

Gil, J. \& Sequera, J. (2018). Expansión de la ciudad turística y nuevas resistencias. El caso de Airbnb en Madrid. Revista de Metodología de Ciencias Sociales, (41), 15-32. https://doi. org/10.5944/empiria.41.2018.22602

Gotham, K. F. (2005). Tourism gentrification: The case of New Orleans' vieux carre (French quarter). The Gentrification Debates, 42(7), 145-176. https://doi. org/10.4324/9781315881096

Horna, K. \& Merantea, M. (2017). Is Home Sharing driving up rents? Evidence from Airbnb in Boston. Journal of Housing Economics, 38, 14-24. https://doi.org/10.1016/j. jhe.2017.08.002

Instituto Nacional de Estadística (España). (2001). Censos de Población y Vivienda 2001. Resultados definitivos. https://www.ine.es/censo2001

Instituto Nacional de Estadística (España). (2011). Censos de Población y Vivienda 2011. https://www.ine.es/censos2011_datos/cen11_datos_inicio.htm

Instituto Nacional de Estadística (España). (2019). Encuesta de ocupación hotelera. Año 2019. www.ine.es/jaxiT3/Tabla.htm?t=2078

Jiménez, J. (1995). Guía de Arquitectura de Cádiz. Consejería de Obras Públicas y Transporte, Sevilla.

Jover, J. \& Díaz-Parra, I. (2019). Gentrification, transnational gentrification and touristification in Seville, Spain. Urban Studies, 1-16. https://doi.org/10.1177/0042098019857585

Mansilla, J. A. (2018). Vecinos en peligro de extinción. Turismo urbano, movimientos sociales y exclusión socioespacial en Barcelona. Pasos - Revista de Turismo y Patrimonio Cultural, 16, 279-296. https://doi.org/10.25145/j.pasos.2018.16.020

Marchena, M. \& Vera, J. F. (1990). Turismo y Desarrollo: Un planteamiento actual. Papers de Turisme, 3, 59-84. https://idus.us.es/xmlui/bitstream/handle/11441/47549/turismo y desarrollo un planteamiento actual.pdf?sequence $=1$

Mato, J. M. (2005). Urbanismo y problemática social en Cádiz. Una aproximación histórica. Trocadero, (17), 163-182. https://doi.org/10.25267/trocadero.2005.i17.08

Milano, C. (2018). Overtourism, malestar social y turismofobia. Un debate controvertido. Pasos - Revista de Turismo y Patrimonio Cultural, 18(3), 551-564. https://doi. org/10.25145/j.pasos.2018.16.041

Morell, M. (2018). Urban tourism via dispossession of oeuvres. Focaal, (82), 35-48. https:// doi.org/10.3167/fcl.2018.820103

Navarrete, D. (2017). Turismo gentrificador en ciudades patrimoniales. Exclusión y transformaciones urbano-arquitectónicas del patrimonio en Guanajuato, México. Revista INVI, 32(89), 61-83. http://revistainvi.uchile.cl/index.php/INVI/article/ view/1019/1321

Ortuño, A. \& Jiménez, J. L. (2019). Economía de plataformas y turismo en España a través de Airbnb. Cuadernos Económicos de ICE, (97), 133-153. https://doi.org/10.32796/ cice.2019.97.6800 
Pavel, F. (2016). El Barrio Alto en Lisboa entre gentrificación, turistificación y derechos de la población. Madrid, ponencia presentada en el Congreso Internacional Contested Cities.

Pons, A., Rullan, O. \& Murray, I. (2014). Tourism capitalism and island urbanization: tourist accommodation diffusion in the Balearics, 1936-2010. Island Studies Journal, 9(2), 239-258. https://www.researchgate.net/publication/268630526

Postma, A. \& Schmuecker, D. (2017). Understanding and overcoming negative impacts of tourism in city destinations: conceptual model and strategic framework. Journal of Tourism Futures, 3(2), 144-156. https://doi.org/10.1108/JTF-04-2017-0022

Rey, S. J., Anselin, L., Li, X., Pahle, R., Laura, J., Li, W. \& Koschinsky, J. (2015). Open geospatial analytics with PySAL. ISPRS International Journal of Geo-Information, 4(2), 815-836. https://doi.org/10.3390/ijgi4020815

Sequera, J. \& Nofre, J. (2018). Shaken, not stirred: New debates on touristification and the limits of gentrification. City, 22(5-6), 834-855. https://doi.org/10.1080/13604813.2 018.1548819

Slater, T. (2009). Missing Marcuse: On gentrification and displacement. City, 12(2-3), 292311. https://doi.org/10.1080/13604810902982250

Troitińo, M. A. (1996). La protección y la recuperación de los centros históricos en España: un reto difícil de las políticas urbanas. En Portugal-España: Ordenación territorial del Suroeste Comunitario. VII Coloquio Ibérico de Geografia (pp. 367-377). Universidad de Extremadura.

Veiga, C., Santos, M. C., Águas, P. \& Santos, J. A. C. (2018). Sustainability as a key driver to address challenges. Worldwide Hospitality and Tourism Themes, 10(6), 662-673. https://doi.org/10.1108/WHATT-08-2018-0054

Villar, A. \& Fernández, A. (2017). Reconstruir la historia del turismo a través de la prensa: la evolución del espacio turístico de Sevilla (1915-2015). Cuadernos Geográficos, 56(1), 290-321. http://hdl.handle.net/11441/59617

Vinuesa, J. (1996). Dinámica de la población urbana en España. Ciudad y Territorio. Estudios Territoriales, 28(107-108), 185-216. https://recyt.fecyt.es/index.php/CyTET/article/ view/84125

Wachsmuth, D. \& Weisler, A. (2018). Airbnb and the rent gap: Gentrification through the sharing economy. Environment and Planning A, 50(6), 1147-1170. https://doi. org/10.1177/0308518X18778038

World Tourism Organization (UNwTO). (2018). 'Overtourism?? Understandingandmanagingurban tourism growth beyond perceptions. UNwTO. https://doi.org/10.18111/9789284420070

\author{
Portales wEB: \\ https://datahippo.org/es \\ www.airbnb.es \\ www.idealista.com \\ www.juntadeandalucia.es/institutodeestadisticaycartografia \\ www.horecacadiz.org \\ www.hotelesdesevilla.com
}

